\title{
A novel and sensitive method for measuring very weak magnetic fields of DA white dwarfs
}

\section{A search for a magnetic field at the $250 \mathrm{G}$ level in 40 Eridani $B^{\star}$}

\author{
J. D. Landstreet ${ }^{1,2}$, S. Bagnulo ${ }^{1}$, G. G. Valyavin ${ }^{3}$, D. Gadelshin ${ }^{3}$, A. J. Martin ${ }^{1,4}$,
}

G. Galazutdinov ${ }^{5,6,3}$, and E. Semenko ${ }^{3}$

\author{
1 Armagh Observatory, College Hill, Armagh, BT61 9DG, Northern Ireland, UK \\ e-mail: [jls; sba]@arm.ac.uk \\ 2 Department of Physics \& Astronomy, University of Western Ontario, London, Ontario N6A 3K7, Canada \\ 3 Special Astrophysical Observatory, Nizhnij Arkhyz, Zelenchukskiy Region, 369167 Karachai-Cherkessian Republic, Russia \\ 4 Astrophysics Group, Keele University, Keele, Staffordshire, ST5 5BG, UK \\ 5 Instituto de Astronomia, Universidad Catolica del Norte, Av. Angamos 0610, Antofagasta, Chile \\ ${ }^{6}$ Pulkovo Observatory, Pulkovskoe Shosse 65, 196140 Saint-Petersburg, Russia
}

Received 28 April 2015 / Accepted 20 June 2015

\begin{abstract}
Context. Searches for magnetic fields in white dwarfs have clarified both the frequency of occurrence and the global structure of the fields found down to field strengths of the order of $500 \mathrm{kG}$. Below this level, the situation is still very unclear.

Aims. We are engaged in a project to find and study the weakest magnetic fields that are detectable in white dwarfs, in order to empirically determine how the frequency of occurrence and the structure of fields present changes with field strength. In this paper we report the successful testing of a very sensitive method of longitudinal field detection in DA white dwarfs. We use this method to carry out an extremely sensitive search for magnetism in the bright white dwarf 40 Eri B.

Methods. The method of field measurement we use is to measure, at high spectral resolution, the polarisation signal $V / I$ of the narrow non-LTE line core in $\mathrm{H} \alpha$ in DA stars. This small feature provides a much higher amplitude polarisation signal than the broad Balmer line wings. We test the usefulness of this technique by searching for a weak magnetic field in 40 Eri B.

Results. One hour of observation of $I$ and $V$ Stokes components of the white dwarf 40 Eri B using ESPaDOnS at the CFHT is found to provide a standard error of measurement of the mean longitudinal magnetic field $\left\langle B_{z}\right\rangle$ of about $85 \mathrm{G}$. This is the smallest standard error of field measurement ever obtained for a white dwarf. The non-detections obtained are generally consistent with slightly less accurate measurements of 40 Eri B obtained with ISIS at the WHT and the Main Stellar Spectrograph at SAO, in order to provide comparison standards for the new method. These further measurements allow us to make a quantitative comparison of the relative efficiencies of low-resolution spectropolarimetery (using most or all of the Balmer lines) with the new method (using only the core of $\mathrm{H} \alpha$ ).

Conclusions. The new method of field detection reaches the level of sensitivity that was expected. It appears that for suitable DA stars, about the same field uncertainties can be reached with ESPaDOnS on the CFHT, in a given integration time, as with FORS on an 8-m telescope, and uncertainties are a factor of two better than with low-resolution spectropolarimetry with other 4-6-m class telescopes. However, even with this extraordinary sensitivity, there is no clear indication of the presence of any magnetic field in 40 Eri B above the level of about $250 \mathrm{G}$.
\end{abstract}

Key words. magnetic fields - polarization - methods: observational - stars: magnetic field - white dwarfs

\section{Introduction}

Megagauss magnetic fields were first discovered in white dwarfs in the 1970 s by detection of broadband circular (and soon afterwards linear) polarisation (Kemp et al. 1970; Angel \& Landstreet 1970a, 1971). It was already understood that such huge fields, and indeed much weaker ones, could be detected by observing the Zeeman effect, for example in Balmer lines (Angel \& Landstreet 1970b), and since the early 1970s the overwhelming majority of magnetic white dwarfs have been discovered using this effect, which in practice is sensitive to fields from some $\mathrm{kG}$ to hundreds of MG in white dwarfs (Landstreet 1992;

* Based in part on observations obtained at the Canada-FranceHawaii Telescope (CFHT) which is operated by the National Research Council of Canada, the Institut National des Sciences de l'Univers of the Centre National de la Recherche Scientifique of France, and the University of Hawaii.
Vanlandingham et al. 2005; Kawka et al. 2007). At present many hundreds of magnetic white dwarfs are known as a result of searches through the thousands of white dwarf spectra obtained by the Sloan Digital Sky Survey (Kepler et al. 2013). It appears that magnetic fields above $\sim 1 \mathrm{MG}$ are present in roughly $10 \%$ of all white dwarfs (Liebert et al. 2003).

The fields of the magnetic white dwarfs are usually measured using the splitting of spectral lines, which provides an estimate of the modulus of the field averaged over the visible stellar hemisphere $\langle B\rangle$. The fields can also be measured using the (usually circular) polarisation signature produced by the Zeeman effect, which can be used to estimate the mean line-of-sight component of the magnetic field, averaged over the visible hemisphere $\left\langle B_{z}\right\rangle$, as reviewed by Landstreet (1992) and Schmidt (2001).

From such measurements it is now known that the fields of white dwarfs range in strength from some tens of $\mathrm{kG}$ up to 
hundreds of MG, with the majority having fields in the range of $1-10^{2} \mathrm{MG}$. These fields are apparently roughly dipolar in structure. The measured field may vary periodically with time as the star rotates (although many white dwarf fields show no sign at all of rotational variations), but it appears that the fields change intrisically only on time scales that are too long to have been detected so far.

The least studied regime of white dwarf magnetism is the low-field end. This is largely due to the difficulty of detecting $\mathrm{kG}$ fields in such faint (usually Johnson $V$ magnitude of 12 or fainter) objects. As a result, only a very small number of magnetic fields below $100 \mathrm{kG}$ have been securely detected (Aznar Cuadrado et al. 2004; Kawka \& Vennes 2012; Landstreet et al. 2012). Even fewer have been modelled in any useful way, so we really do not yet even know if the weak fields found in a few white dwarfs are similar in structure to the common stronger fields or if they represent a significantly different phenomenon.

This deficiency in our knowledge of the weak field end of the field strength distribution is important because we do not clearly understand at present how the fields of white dwarfs arise. It has been proposed that they may be the fossil remmnants of magnetism inherited from an earlier stage of evolution, for example from the main sequence (Landstreet 1992). Alternatively, the fields of white dwarfs may have a more specific origin, such as being produced during the mergers of close binary systems (Valyavin \& Fabrika 1999; Tout et al. 2008; Wickramasinghe et al. 2014).

There are not many kinds of information about white dwarf magnetism that really provide useful tests and constraints on theoretical ideas about how magnetism arises in white dwarfs. The distribution of magnetic fields strengths over the white dwarf population is one kind of valuable information, but the lowfield end of this distribution is very poorly known. Another kind of constraint is provided by detailed models of individual white dwarf magnetic fields. Modelling has proved rather difficult at the high-field end of the distribution, but models of field structure in the 1-30 MG range are fairly well developed (Wickramasinghe 2001). At the lowest fields, the lack of suitable candidate stars to model as well as the lack of detailed and repeated observations of the tiny number of known, extremely weak-field white dwarfs has meant that at present it is really not even clear whether the weakest magnetic fields are similar in structure to larger fields in other magnetic white dwarfs.

We have started a project to try to obtain the necessary observational data to clarify both the frequency of very weak magnetic fields in white dwarfs and the structure of fields found. Because the fields we seek are at the limit of detectability, we have looked for new ways to improve the sensitivity of our measurements. In this work, we describe a novel technique to search for and study the weakest DA white dwarf fields. We describe the new technique in detail, and test it on the bright DA white dwarf 40 Eri B. We also report several conventional but still very precise measurements of the field of 40 Eri B made to corroborate measurements obtained with the new method, and to allow a direct comparison of the field sensitivity limits obtained with various methods. These measurements confirm the exceptional field sensitivity of our new method, and the non-detection of a field in 40 Eri B.

\section{A new technique for weak field detection in white dwarfs}

Detection of magnetic fields in white dwarfs is done using various aspects of the Zeeman effect (and its large-field extensions).
In the presence of a field, single spectral lines split into multiple components, and this splitting can be observed directly in simple intensity (Stokes $I$ ) spectra if it is large enough. Measuring the separation of the outer components provides a measurement of the mean field modulus $\langle B\rangle$, the magnitude of the field averaged over the visible stellar hemisphere.

In addition, the component(s) of the split line that shift systematically to longer and shorter wavelengths from the unperturbed wavelength (the $\sigma$ components) become circularly polarised in opposite senses if the field has a net component along the line of sight. This causes the mean position of the spectral line to be at slightly different wavelengths as observed in right and left circularly polarised light. This slight difference between the mean positions of a spectral line as seen in the two polarisations can conveniently be measured by forming the difference between the two polarised spectra (Stokes $V$ ), as will be discussed below. Measuring this $V$ difference spectrum makes possible the deduction of the mean line-of-sight component of the field $\left\langle B_{z}\right\rangle$.

Because almost all white dwarfs are quite faint (generally fainter than magnitude 12 or 13), most white dwarf spectroscopy is done at rather low resolving power $R$, typically 1000-2000, and often with fairly low signal-to-noise ratio, of the order of 20 or 30. Under these conditions, Zeeman splitting can be detected in the unresolved cores of the broad (roughly 1-200 $\AA$ wide) Balmer lines if a field of at least several hundred $\mathrm{kG}$ is present. Most of the $\sim 10^{3}$ magnetic white dwarfs now known have been identified in this manner (e.g. Kepler et al. 2013).

However, it is well known that the $\mathrm{H} \alpha$ line in many DA white dwarfs possesses a deep and narrow non-LTE line core. Observations of white dwarfs at high spectral resolution, for example in the context of the ESO SPY search for SN Ia progenitors (Koester et al. 2001, 2009) can resolve this narrow feature. Because the full width of this line core is only about $1.5 \AA$, much smaller line splitting can be detected than is visible at low resolution, and fields as small as about 20 or $30 \mathrm{kG}$ can be identified and measured. The very weak-field magnetic white dwarf WD2105-820 (Landstreet et al. 2012) was first identified as a candidate magnetic white dwarf by Koester et al. (1998) on the basis of slight broadening of the $\mathrm{H} \alpha$ core by an amount which suggested the presence of a field with $\langle B\rangle \sim 40 \mathrm{kG}$.

To search for still weaker magnetic fields, spectropolarimetry is essential. The small difference in radial velocity between spectral lines as seen in the two senses of polarisation can be measured by detecting the tiny differences in line wing depth of opposite sign in the two line wings. This measurement is usually made with a low resolution spectropolarimeter. By combining the signals from several Balmer lines (or He lines), uncertainties of $\left\langle B_{z}\right\rangle$ can be as low as a few hundred $\mathrm{G}$, making possible the detection of longitudinal fields as small as a few kG. Fields of $\left\langle B_{z}\right\rangle \sim 3-10 \mathrm{kG}$ have been discovered and measured with low-resolution circular spectropolarimetry using FORS1 at ESO (Aznar Cuadrado et al. 2004; Landstreet et al. 2012).

Several very efficient high-resolution spectropolarimeters are now available on intermediate aperture telescopes (ESPaDOnS on the CFHT, Narval on the TBL at Pic-du-Midi, and HARPSpol on the La Silla 3.6-m telescope). These instruments have never, so far as we are aware, been used for magnetic field measurement of white dwarfs. An important reason that such instruments have not been used for white dwarf field measurements is that all these instruments include cross-dispersed echelle spectrographs with rather short orders, and are fibrefed from fibres that originate after the polarisation optics. Any 


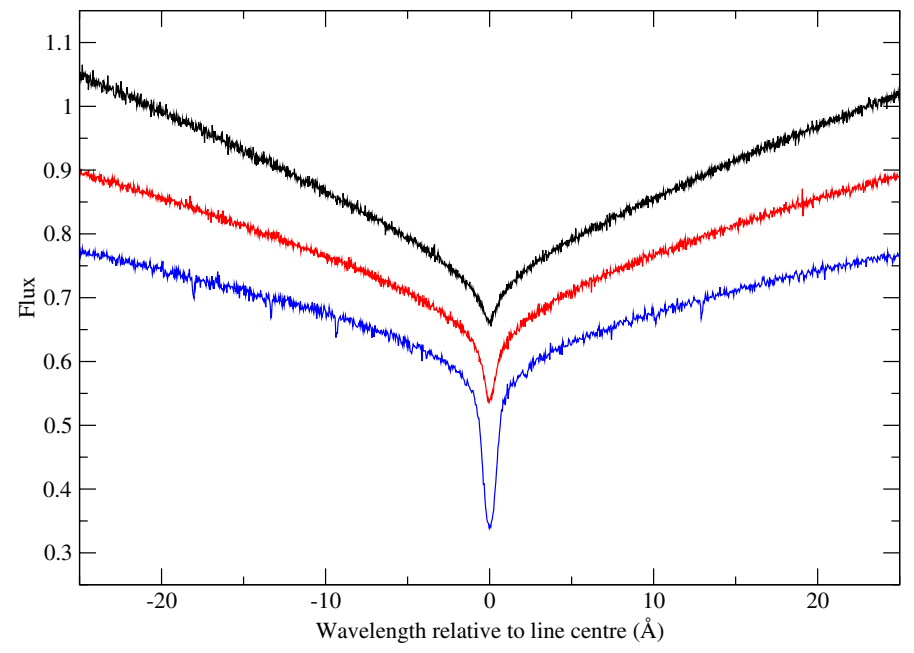

Fig. 1. Balmer lines $\mathrm{H} \alpha$ (bottom), $\mathrm{H} \beta$ (middle), $\mathrm{H} \gamma$ (top) from a typical ESPaDOnS spectrum of 40 Eri B. The continua for all lines are normalised to 1.0 in the far wings. The upper two spectra have been shifted upward for clarity by $0.2(\mathrm{H} \beta)$ and $0.3(\mathrm{H} \gamma)$. The unique shape of the core of $\mathrm{H} \alpha$ is clear from this figure.

broad-band polarisation signal is likely to be contaminated by instrumental polarisation due to seeing and guiding fluctuations on the entrance aperture, to flexures in the Cassegrain polarimetric unit, and to transmission variations in the fibres, as the telescope moves. For these reasons, any broadband polarimetric signal present in the light is usually set to zero during reduction. Polarisation is normally measured with such instruments across spectral lines that are very narrow compared to the length of an order, relative to an assumed unpolarised continuum. As a consequence, the tiny broad-band circular polarisation (comparable in scale to whole orders) that may be present in the broad wings of $\mathrm{H}$ lines in white dwarfs cannot generally be measured reliably with high-resolution spectropolarimeters.

However, DA white dwarfs with $T_{\text {eff }}$ in the range $20000 \geq$ $T_{\text {eff }} \geq 8000 \mathrm{~K}$ do possess one spectral feature that is well suited to polarimetric measurement with such high-resolution instruments: the sharp and deep non-LTE core of $\mathrm{H} \alpha$. This feature is illustrated in Fig. 1, which compares the core profiles of $\mathrm{H} \alpha, \mathrm{H} \beta$ and $\mathrm{H} \gamma$ of the DA3 white dwarf 40 Eri B as observed with a resolving power of $R=65000$ (see below). The uniquely sharp and deep nature of the core of $\mathrm{H} \alpha$ is clear. $\mathrm{H} \beta$ also has a sharp core, but this core is substantially shallower than that of $\mathrm{H} \alpha$ and so the amplitude of a circular polarisation signal from the $\mathrm{H} \beta$ core would be several times smaller than that of $\mathrm{H} \alpha$. Note also that the sharp core of $\mathrm{H} \alpha$ weakens above $T_{\text {eff }} \sim 20000 \mathrm{~K}$, and disappears around $30000 \mathrm{~K}$.

It is of course not obvious that a single spectral feature can provide a useful level of field measurement precision, since the polarisation measurement uses only about $2 \AA$ of the entire spectrum, while a normal white dwarf $\left\langle B_{z}\right\rangle$ measurement uses nearly the full width of several Balmer lines, and thus exploits up to half the stellar flux observed with the instrument. Clearly the loss of information by using a tiny fraction of the flux is severe. To compensate, however, the slope of the line wings of the line core may be 20 or 30 times larger than the slope of the broad Balmer line wings in a white dwarf. Because of this, the polarisation signal in the wings of the non-LTE line core is 20 or 30 times larger than the signal in the extended line wings, and thus a measurement with $S / N \sim 30$ times lower (and a flux $\sim 10^{3}$ times smaller) than is required to detect the broad line wing polarisation may make possible an accurate $\left\langle B_{z}\right\rangle$ measurement using a high-resolution spectrograph.

We conclude that measurement of $\left\langle B_{z}\right\rangle$ using the core of $\mathrm{H} \alpha$ might provide a substantial improvement in field measurement uncertainty relative to measurements made by a conventional method. It is clearly important to determine experimentally whether this novel field measurement technique is actually sensitive enough to compete with conventional low-resolution, full-spectrum spectropolarimetry.

The one published set of white dwarf observations employing the non-LTE core of $\mathrm{H} \alpha$ that we know of was a series of measurements in 1995 of the field of 40 Eri B using the polarimeter on the high resolution Main Stellar Spectrograph (MSS) at the Special Astrophysical Observatory (SAO; Fabrika et al. 2003). The authors report detection of a variable field of semi-amplitude $2.3 \mathrm{kG}$ varying with a period of a few hours. The reported measurements, which lasted $11 \mathrm{~min}$ each, had uncertainties of typically 1.5 to $2 \mathrm{kG}$, so that the reported field could only be detected by combining the individual meaurements. However, the general potential of using the $\mathrm{H} \alpha$ core to study white dwarf fields was not clearly recognised, and this measurement technique was not pursued further.

Modern spectropolarimeters have substantially higher efficiency than the SAO spectrograph did in the 1990s. Comparison of the shape of the non-LTE line core of a few white dwarfs with the shape of individual lines in rotionally broadened magnetic main sequence A and B stars that have been previously measured with high resolution suggested that an uncertainty of the order of $10^{2} \mathrm{G}$ could be obtained for a white dwarf of magnitude 9 in one hour. Clearly an experiment is called for to discover what level of precision in $\left\langle B_{z}\right\rangle$ can be achieved in practice for DA white dwarfs with a modern high-resolution spectrograph.

\section{ESPaDOnS observations and data reduction}

We have tested this method of white dwarf field measurement using the high-resolution spectropolarimeter ESPaDOnS at the Canada-France-Hawaii Telescope (CFHT), located on Mauna Kea, Hawaii. This instrument uses a set of three Fresnel rhombs to make possible measurement of all four Stokes parameters. The polarisation analyser, mounted on the telescope, produces a double beam that is fibre-fed into the entrance aperture of a cross-dispersed spectrograph having $R \approx 65000$. The output spectra are recorded on a single CCD chip, and subsequently reduced using the Libre-Esprit reduction programme.

We have tested the potential of high-resolution $\mathrm{H} \alpha$ spectropolarimetry for field measurement by observing 40 Eri B, the brightest easily observed white dwarf in the sky. 40 Eri B is a DA3 star, with $T_{\text {eff }}=17100 \mathrm{~K}$ and $\log g=7.95$ (Giammichele et al. 2012). It is thus a rather typical white dwarf with an H-rich atmosphere, except for its particularly close distance of about 5 pc. Furthermore, as noted above, a variable magnetic field of $\left\langle B_{z}\right\rangle \sim 2 \mathrm{kG}$ was been reported in the star by Fabrika et al. (2003).

Because Fabrika et al. (2003) reported a rotation period of about $5 \mathrm{~h}$, we used 1-h observing sequences (one sequence = four observations with alternating waveplate positions). Such long measurements would smear the $\left\langle B_{z}\right\rangle$ measurements over only about 0.2 cycle, which should allow easy detection of the $2 \mathrm{kG}$ field reported by the SAO team if our precision goal was met. Two one-hour long sequences of observations were made on each of three different nights, one hour apart.

Each set of four subexposures produces an $I(\lambda)$ and a $V(\lambda)$ spectrum covering approximately $3800 \AA$ to $1.04 \mu \mathrm{m}$ in about 
40 orders. The reduction process also creates two different null spectra and a table of pixel-by-pixel uncertainties $\sigma_{i} . \mathrm{H} \alpha$ is present in two orders (as is $\mathrm{H} \beta$ ). We find that a sharp nonLTE line core is also present in much weaker form in $\mathrm{H} \beta$, but completely absent from higher Balmer lines. Thus almost all the signal worth working with is in two orders, each of which covers the core and a part of the broad wings of $\mathrm{H} \alpha$.

The value of $\left\langle B_{z}\right\rangle$ is evaluated in these two orders (and in the two orders that span the core of $\mathrm{H} \beta$ ) with a small FORTRAN 90 programme that evaluates numerically the usual integral (Donati et al. 1997),

$\left\langle B_{z}\right\rangle=-2.14 \times 10^{12} \frac{\int v V(v) \mathrm{d} v}{\lambda_{0} c \int\left(I_{\text {cont }}-I(v)\right) \mathrm{d} v}$

where the intensity $I$ and circular polarisation $V$ are expressed as function of velocity $v$ relative to the central wavelength of the line $\lambda_{0}$, and $c$ is the speed of light (Donati et al. 1997). The Landé factor is assumed to be 1.0 for the Balmer lines. This expression is exactly equivalent to evaluating the separation between the centroids of the right and left circularly polarised line core profiles, and $I_{\text {cont }}$ is simply the upper boundary of the line cores used in evaluating the centroid positions, so its level is chosen to provide the highest possible $\mathrm{S} / \mathrm{N}$ for the separation measurement.

With a discretely sampled spectrum, the integral is conveniently approximated by a summation over the line:

$\left\langle B_{z}\right\rangle \approx-2.14 \times 10^{12} \frac{\sum_{\text {line }} v_{i} V_{i}}{\lambda_{0} c \sum_{\text {line }}\left(I_{\text {cont }}-I_{i}\right)}$,

where the summation extends over the pixels in the spectral line. Examining the equation in summation form, it is clear, from the general expression for the uncertainty of a sum of terms $i$ with known Gaussian errors, that the uncertainty of $\left\langle B_{z}\right\rangle$ is given by

$\sigma_{\left\langle B_{z}\right\rangle} \approx-2.14 \times 10^{12} \frac{\left[\sum_{\text {line }}\left(v_{i} \sigma_{i}\right)^{2}\right]^{1 / 2}}{\lambda_{0} c \sum_{\text {line }}\left(I_{\text {cont }}-I_{i}\right)}$,

where $\sigma_{i}$ is the uncertaintiy assigned to $V_{i}$ by the Libre-Esprit reduction programme.

The integration window used basically coincides with the line core, and was chosen (after some experimentation) to be $1.6 \AA$ in full width. The results from the two orders (which represent simultaneous and essentially independent measurements of the field) are averaged. The (averaged) field strength $\left\langle N_{z}\right\rangle$ resulting from one of the null spectra (which should be zero within the uncertainty) was also computed as a check on the reasonableness of the measurement and particularly of the computed uncertainty $\sigma_{\left\langle B_{z}\right\rangle}$.

Figure 2 shows that the polarisation signature that we are searching for occurs in the line core of $\mathrm{H} \alpha$. We have tested our field measurement method further by using it to detrmine the value of $\left\langle B_{z}\right\rangle$ from ESPaDOnS polarised spectra of several magnetic Ap and Bp stars. In fact, the line cores of the Balmer lines in magnetic Ap/Bp stars of low $v \sin i$ are quite similar to those of white dwarfs: $\mathrm{H} \alpha$ has a narrow and deep core, $\mathrm{H} \beta$ has a shallower sharp core, and the sharp cores quickly vanish as one moves up the Balmer series. We have measured $\left\langle B_{z}\right\rangle$ in spectra of four such stars using $\mathrm{H} \alpha$, and compare it to the field values measured from the same spectra using least squared deconvolution (LSD) as described by Landstreet et al. (2008).

The results of this comparison are shown in Table 1. Several conclusions may be drawn from these results. First, it is clear that $\left\langle B_{z}\right\rangle$ measurements using the core of $\mathrm{H} \alpha$ are practical and yield

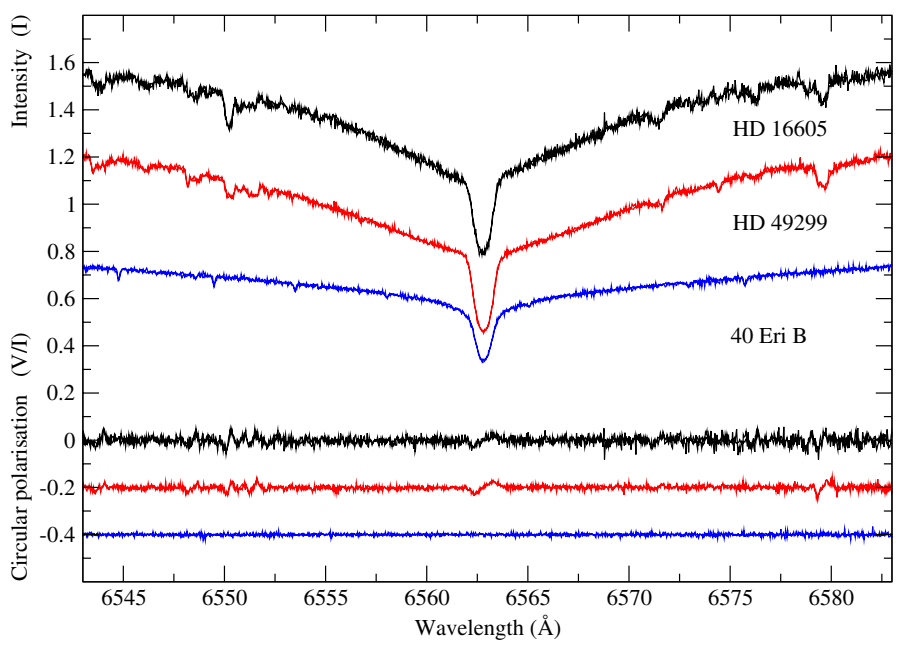

Fig. 2. The deep $I \mathrm{H} \alpha$ line core and a typical observed $V / I$ circular polarisation spectrum of 40 Eri B is compared to similar line cores and polarisation spectra of two magnetic Ap stars, HD 16605 and HD 49299, all observed with ESPaDOnS. The two magnetic Ap stars both have magnetic fields of $\left\langle B_{z}\right\rangle \sim 2.5 \mathrm{kG}$ at the time of observation. The $V / I$ spectrum of 40 Eri $\mathrm{B}$ shows no sign of a magnetic signature at a level several times smaller than the signals clearly visible in the $V / I$ spectra of the Ap stars. All $I$ spectra are normalised to 1.0 in the continuum, shifted vertically by +0.3 (HD 42999) or +0.6 (HD 16605); $V / I$ spectra are shifted downward from 0.0 by -0.2 (HD 49299 ) or -0.4 (40 Eri B).

Table 1. $\left\langle B_{z}\right\rangle$ measured in magnetic sharp-line Ap/Bp stars with our $\mathrm{H} \alpha$ method, compared to $\left\langle B_{z}\right\rangle$ measured from the same spectra using LSD.

\begin{tabular}{cccc}
\hline \hline Star & MJD & $\begin{array}{c}\mathrm{H} \alpha\left\langle B_{z}\right\rangle \\
(\text { Gauss })\end{array}$ & $\begin{array}{c}\text { LSD }\left\langle B_{z}\right\rangle \\
\text { (Gauss) }\end{array}$ \\
\hline HD 16605 & 53570.58 & $-1380 \pm 135$ & $-2205 \pm 29$ \\
HD 49299 & 56293.42 & $-1935 \pm 74$ & $-2530 \pm 49$ \\
HD 133652 & 56437.35 & $+842 \pm 72$ & $+1330 \pm 18$ \\
HD 318107 & 54552.62 & $+4340 \pm 110$ & $+4900 \pm 45$ \\
\hline
\end{tabular}

essentially the expected results. Secondly, the $\left\langle B_{z}\right\rangle$ values resulting from the $\mathrm{H} \alpha$ core are somewhat different from those obtained with LSD. This is a very common situation; each specific instrumental system used for measuring $\left\langle B_{z}\right\rangle$ yields, in general, mildly different results than those obtained from other systems (see the discussion in Sect. 4 of Landstreet et al. 2014). Finally, it appears that the field as measured using $\mathrm{H} \alpha$ may be slightly weaker than from other methods, as this is the case for all four measurements in the table. However, no test has been possible yet to directly compare $\left\langle B_{z}\right\rangle$ measurements obtained using the core of $\mathrm{H} \alpha$ with those obtained by observing the wider Balmer line wings at low resolution. We will assume until such tests are carried out that the sensitivity of this new method is essentially the same as other methods.

The particulars of the observations of 40 Eri B and the resulting field strength $\left\langle B_{z}\right\rangle$, null field $\left\langle N_{z}\right\rangle$, and uncertainty $\sigma$ are shown in Table 2. Each observation lasted (with readout) $1.0 \mathrm{~h}$. $\left\langle B_{z}\right\rangle$ was evaluated for $\mathrm{H} \beta$ as well as $\mathrm{H} \alpha$, but the corresponding uncertainties, around $450-600 \mathrm{G}$, are so large that we did not combine them with the $\mathrm{H} \alpha$ data or present them here. Within their uncertainties, the $\mathrm{H} \beta$ results are fully consistent with the $\mathrm{H} \alpha$ measurements.

The essential information derived from these spectra is shown in Fig. 2. This figure displays a single $I$ spectrum of 40 Eri B, and the corresponding $V / I$ spectrum (all the $I$ and 
Table 2. Magnetic field measurements of 40 Eri B made with ESPaDOnS at the CFHT.

\begin{tabular}{cccc}
\hline $\begin{array}{c}\text { Date } \\
\text { yr-mo-day }\end{array}$ & MJD & $\begin{array}{c}\left\langle B_{z}\right\rangle \\
\text { (Gauss) }\end{array}$ & $\begin{array}{c}\left\langle N_{z}\right\rangle \\
\text { (Gauss) }\end{array}$ \\
\hline $2014-09-12$ & 56912.502 & $174 \pm 91$ & $41 \pm 91$ \\
& 56912.543 & $-14 \pm 88$ & $-91 \pm 88$ \\
$2014-09-14$ & 56914.579 & $-90 \pm 83$ & $-9 \pm 83$ \\
& 56914.619 & $-44 \pm 83$ & $107 \pm 83$ \\
$2014-09-16$ & 56916.576 & $212 \pm 88$ & $147 \pm 88$ \\
& 56916.617 & $77 \pm 91$ & $26 \pm 91$ \\
\hline
\end{tabular}

$V / I$ spectra are essentially identical). For comparison, we also display the $I$ and $V / I$ ESPaDOnS spectra of the A0p-A1p main sequence stars HD 16605 and HD 49299, which at the times of observation both had magnetic fields of $\left\langle B_{z}\right\rangle \approx-2.5 \mathrm{kG}$. In spite of being main sequence stars, these stars have $\mathrm{H} \alpha$ line cores that are rather similar to that of 40 Eri B, although the broad line wings of the Ap star clearly have larger slopes than those of the white dwarf. The spectra of the two main sequence stars illustrate how the $V / I \mathrm{H} \alpha$ line core spectrum of a magnetic star with a $\mathrm{kG}$ field would appear, and also shows clearly how the line core signal is enormously strengthened relative to the (invisibly small) signal in the broad $\mathrm{H} \alpha$ line wings by the sharpness of the narrow core.

It is clear from the figure and from Table 2 that the $V / I$ spectrum of 40 Eri B shows no significant sign of a magnetic signature, and that any field present must be at least a factor of five or so smaller than that of the Ap stars, in agreement with the results of the numerical evaluation of $\left\langle B_{z}\right\rangle$. We conclude that the ESPaDOnS field measurements put a probable limit on any slowly-varying (time scale of hours or more) field $\left\langle B_{z}\right\rangle$ in 40 Eri B of roughly $250 \mathrm{G}$, at least at the times when we observed.

It is important to consider the limitations of such measurements that may be introduced by the rotation of a white dwarf. Because white dwarfs are small (with radii of the order of $10^{4} \mathrm{~km}$ ) the normal upper limits derived for projected rotation velocity, typically about $30 \mathrm{~km} \mathrm{~s}^{-1}$, lead to lower limits on rotation periods of the order of half an hour (Heber et al. 1997). Furthermore, a few strongly magnetic white dwarfs are actually observed to have rotation periods of less than an hour (Brinkworth et al. 2013). Thus a measurement such as ours, lasting an hour or more, might in some cases last longer than the rotation period of the star. If the $\left\langle B_{z}\right\rangle$ field measurement reverses sign during rotation, the measured field could average out to a substantially smaller value than would be observed by measurments lasting only a few minutes.

This probably not a major measurement limitation in the specific case of our measurements of 40 Eri B. Heber et al. (1997) have shown that the rotation period of 40 Eri $\mathrm{B}$ is longer than about $2 \mathrm{~h}$, so that each single measurement of the star is at most about half a rotation. Thus some of our measurements at least should have observed mainly one magnetic hemisphere of any dipole-like field, and should have detected a field with about the sensitivity given in Table 2. However, this potential limitation of white dwarf field measurements based on long integrations should be kept in mind.

It is worthwhile noting one further important strength of the high-resolution $\mathrm{H} \alpha\left\langle B_{z}\right\rangle$ measurement. As already pointed out by Fabrika et al. (2003), observation with a high-resolution spectrograph has the advantage that the $I$ profile of the non-LTE core of $\mathrm{H} \alpha$ can also be studied, and thus the value (or an upper limit) of $\langle B\rangle$, measured at the same time as the $\left\langle B_{z}\right\rangle$ measurement, can be obtained from the observations. The resulting measurement pairs can be very useful for initial field structure modelling.

\section{Field measurements with the SAO Main Stellar Spectrograph}

To test the correctness of the high-resolution $\mathrm{H} \alpha$ measurements, and for a comparison of conventional measurement methods of white dwarf field strength with measurement using the $\mathrm{H} \alpha$ core, we report measurements of the magnetic field of 40 Eri B made using two lower-resolution spectropolarimeters, the MSS at the SAO, and ISIS at the WHT.

Measurements of Zeeman polarisation of 40 Eri B were carried out with the MSS, permanently installed at the 6-m telescope (BTA) at the Special Astrophysical Observatory of the Russian Academy of Sciences, located on Mt. Pastukhova near Zelenchukskaya in the Karachai-Cherkessian Republic, Russia. The MSS is a longslit spectrograph equipped with a circular polarisation analyser combined with an image slicer (Chountonov 2004). The analyser has a rotatable quarter-wave plate that is able to take two fixed positions corresponding to the angles $-45 \mathrm{deg}$ and $+45 \mathrm{deg}$ relative to the principal axes of the birefringent crystal. More details about the instrument and observational technique can be found in Joshi et al. (2012) and Kudryavtsev et al. (2006). The MSS spectropolarimeter exploits two regimes of spectral resolution: $R=15000$ and $R=6000$. We used both of the regimes in observations of 40 Eri B. In contrast to the previous observations of this white dwarf with this instrument (Fabrika \& Valyavin 1999), the image slicer now employed provides the MSS with much higher stability, which makes it possible to carry out measurement of stellar longitudinal fields with uncertainties as low as $30 \mathrm{G}$ (Kudryavtsev et al. 2006).

All observations of 40 Eri B were conducted following the standard procedures that we usually use (for instance Valyavin et al. 2005). A polarisation observation consists of a series of paired exposures obtained at two orthogonal orientations of the quarter-waveplate. The data reduction is also standard.

We usually make longitudinal field measurements of white dwarfs by measurement of the displacement between the positions of spectral lines in the spectra of opposite circular polarisations. For determination of the line profile displacement we used a technique based on estimates of the autocorrelation function of Zeeman shifted spectral features using Fourier transforms (Scargle 1989). This technique is insensitive to uncertainties in the continuum determination (Klochkova et al. 1996), a feature that is important in observations with the MSS. Comparison of the sensitivity of this method with other methods (Monin et al. 2002) and our own experience make this method preferable in low or moderate resolution observations of magnetic white dwarfs with the MSS. However, the high-resolution (R15000) observations of $40 \mathrm{EriB}$ reported here, when reduced with this method, were found to exhibit artificially small error bars. Due to this problem the high-resolution estimate of the field of 40 Eri B measured with R15000 was also made by the regression method, which demonstrated much more robust accuracy in measurements of the narrow central $\mathrm{H} \beta$ profile. This particular observation can be compared fairly directly with the data from the CFHT.

The reduced magnetic field measurements of 40 Eri B obtained with the MSS are presented in Table 2. The columns are self-explanatory. The first observation, using only $\mathrm{H} \beta$, was obtained with $R=15000$, while the rest were obtained at 


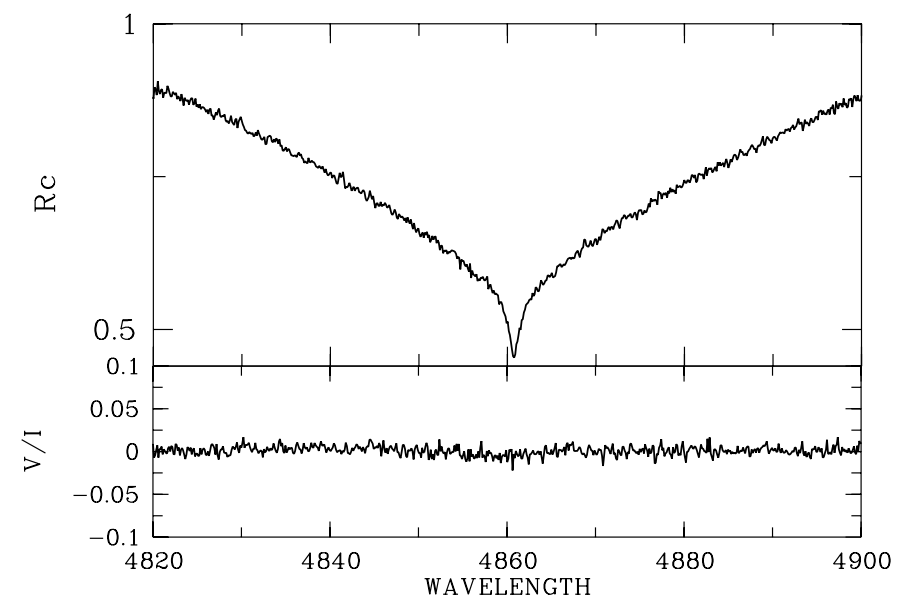

Fig. 3. The intensity $(I)$ and circular polarisation $(V / I)$ spectra of the $\mathrm{H} \beta$ line observed with the MSS at SAO with $R=15000$. The weak sharp core of $\mathrm{H} \beta$ (also illustrated in Fig. 1) is clearly resolved in these data. No sign of any magnetic polarisation signature is seen at line centre.

$R=6000$. Figure 3 shows the $I$ and $V / I$ spectra obtained with the $\mathrm{R} 15000$ resolution. The sharp core is clearly visible at this resolution. However, the fact that the core of $\mathrm{H} \alpha$ is about three times deeper than that of $\mathrm{H} \beta$, and slightly narrower, means that the $\mathrm{S} / \mathrm{N}$ is $\mathrm{H} \alpha$ is about four times higher than that of $\mathrm{H} \beta$ for $\left\langle B_{z}\right\rangle$ measurements made with both lines, as found with the ESPaDOnS data described above.

These observations were all taken within a span of five nights. It is possible that 40 Eri B rotates slowly enough that these measurements all sample one hemisphere. In this case it is meaningful to average all the field measurements together. The mean field measured using all five measurements (R6000 and $\mathrm{R} 15000$ together) is $\left\langle B_{z}\right\rangle=+340 \pm 202 \mathrm{G}$. Averaging only the four R6000 data together yields $\left\langle B_{z}\right\rangle=+119 \pm 283 \mathrm{G}$.

None of these observations, taken individually or together, provide strong evidence for the presence of a field. From these measurements, it does not appear that the $\left\langle B_{z}\right\rangle$ field of 40 Eri B observed by us exceeds perhaps $1 \mathrm{kG}$. This is fully consistent with the measurements made with ESPaDOnS, but is inconsistent with the earlier report of a field of $\left\langle B_{z}\right\rangle \sim 3-5 \mathrm{kG}$ varying sinusoidally with a period of about $5 \mathrm{~h}$ (Fabrika et al. 2003). If a field of that strength were present in 40 Eri B, we would expect that we should have detected it at the level of 5-6 $\sigma$ in at least two or three of the recent observations. It now appears that the old SP-124 spectropolarimeter used for most of the earlier observations of 40 Eri B had larger uncertainties than was recognised at the time, and so we no longer consider the field reported by Fabrika et al. (2003) to be securely detected. This problem has already been briefly discussed in the context of observation of the weak-field white dwarf WD0009+501 (Valyavin et al. 2005).

\section{Field measurements with ISIS at the WHT}

Magnetic observations of 40 Eri B were carried out during a mission with the Intermediate dispersion Spectrograph and Imaging System (ISIS) at the William Herschel Telescope (WHT) at the Observatorio del Roque de los Muchachos on La Palma in the Canaries. This is an intermediate resolution spectrograph that can be used as a spectropolarimeter with the addition of a rotatable quarter-wave plate above the slit, followed by a Savart plate (a linearly polarising beam-splitter) immediately below the slit.
Table 3. Magnetic field measurements of 40 Eri B made with the MSS at the SAO.

\begin{tabular}{ccccc}
\hline $\begin{array}{c}\text { Date } \\
\text { yr-mo-day }\end{array}$ & MJD & $\begin{array}{c}t_{\exp } \\
(\mathrm{s})\end{array}$ & $\begin{array}{c}\left\langle B_{z}\right\rangle \\
\text { (Gauss) }\end{array}$ & $\begin{array}{c}\text { Lines } \\
\text { used }\end{array}$ \\
\hline $2014-10-09$ & 56938.944 & 3600 & $+573 \pm 290$ & $\mathrm{H} \beta$ \\
$2014-10-10$ & 56940.042 & 7200 & $-46 \pm 638$ & $\mathrm{H} \beta-\mathrm{H} \delta$ \\
$2014-10-11$ & 56941.021 & 9600 & $-208 \pm 434$ & $\mathrm{H} \beta-\mathrm{H} \delta$ \\
$2014-10-12$ & 56942.000 & 7200 & $898 \pm 569$ & $\mathrm{H} \beta-\mathrm{H} \delta$ \\
$2014-10-14$ & 56944.000 & 3600 & $-43 \pm 780$ & $\mathrm{H} \beta-\mathrm{H} \delta$ \\
\hline
\end{tabular}

The wave-plate axis is set alternately at $+45 \mathrm{deg}$ and $-45 \mathrm{deg}$ to the principal axes of the Savart plate.

Observations were carried out using the R600B grating, covering a useful spectral range of 3600 to $5200 \AA$. The slit was set to 1 arcsec, leading to a slit image width of about $1.8 \AA$, and a resolving power of $R \approx 2450$. With these instrument settings the weak sharp core of $\mathrm{H} \beta$ is not resolved.

During the three-night run there were frequent thin clouds. The seeing was quite variable, between 0.4 and 2 arcsec, and integration times were adjusted on the fly to compensate.

The three observations were reduced using the same regression technique used to reduce spectropolarimetric observations made with the (rather similar) FORS spectropolarimeter at ESO. A correlation plot is created between the local slope of the $I$ profile and the local value of $V / I$ in the circular polarisation spectrum, and the magnetic field is deduced from slope of the best fit correlation line using the expression

$V(\lambda)=-g_{\mathrm{eff}} C_{Z} \lambda^{2} \frac{\mathrm{d} I(\lambda)}{\mathrm{d} \lambda}\left\langle B_{z}\right\rangle$

where $C_{Z}=e / 4 \pi m_{\mathrm{e}} c^{2}=4.67 \times 10^{-13} \AA^{-1} \mathrm{G}^{-1}$. This method is discussed in considerable detail by Bagnulo et al. (2002, 2012).

The reduction technique is illustrated in Fig. 4. In the upper panel, the uncalibrated stellar spectrum is shown in black in arbitrary units. At the top and bottom of this panel, green bands indicate the regions of spectral lines used for the reduction. The red noise band centred on 0 is the observed $V / I=P_{V}$ spectrum. The blue band below centred on -1 is a null spectrum $N_{V}$ which should show no Zeeman polarisation signal, but should have the same noise behaviour as $P_{V}$. Within the null spectrum, a continuous light blue band indicates the uncertainty of each pixel in the spectra.

The two lower panels show the correlation diagrams from which the field strength $\left\langle B_{z}\right\rangle$ is deduced, constructed by plotting all the point pairs of $P_{V}$ against the corresponding local values of $-4.67 \times 10^{-13} \lambda^{2}(1 / I)(\mathrm{d} I / \mathrm{d} \lambda)$. The left panel is the correlation diagram for $P_{V}$, the right the corresponding diagram for $N_{V}$. The horizontal lines are the fits to the correlation diagrams, and in this case neither exhibits significant slope, indicating that $\left\langle B_{z}\right\rangle$ and $\left\langle N_{z}\right\rangle$ are both zero within uncertainties.

The results of the three observations are shown in Table 4. None of the measurements of $\left\langle B_{z}\right\rangle$ is significantly different from zero. With the uncertainties obtained, it again seems clear that a field of about $2 \mathrm{kG}$ should have been detected.

\section{Comparison of methods}

The data sets above (and comparable data available from white dwarf magnetic observations made with FORS1) make it possible to understand which of the four spectropolarimeters are best adapted for searching for the very weakest fields that may be 

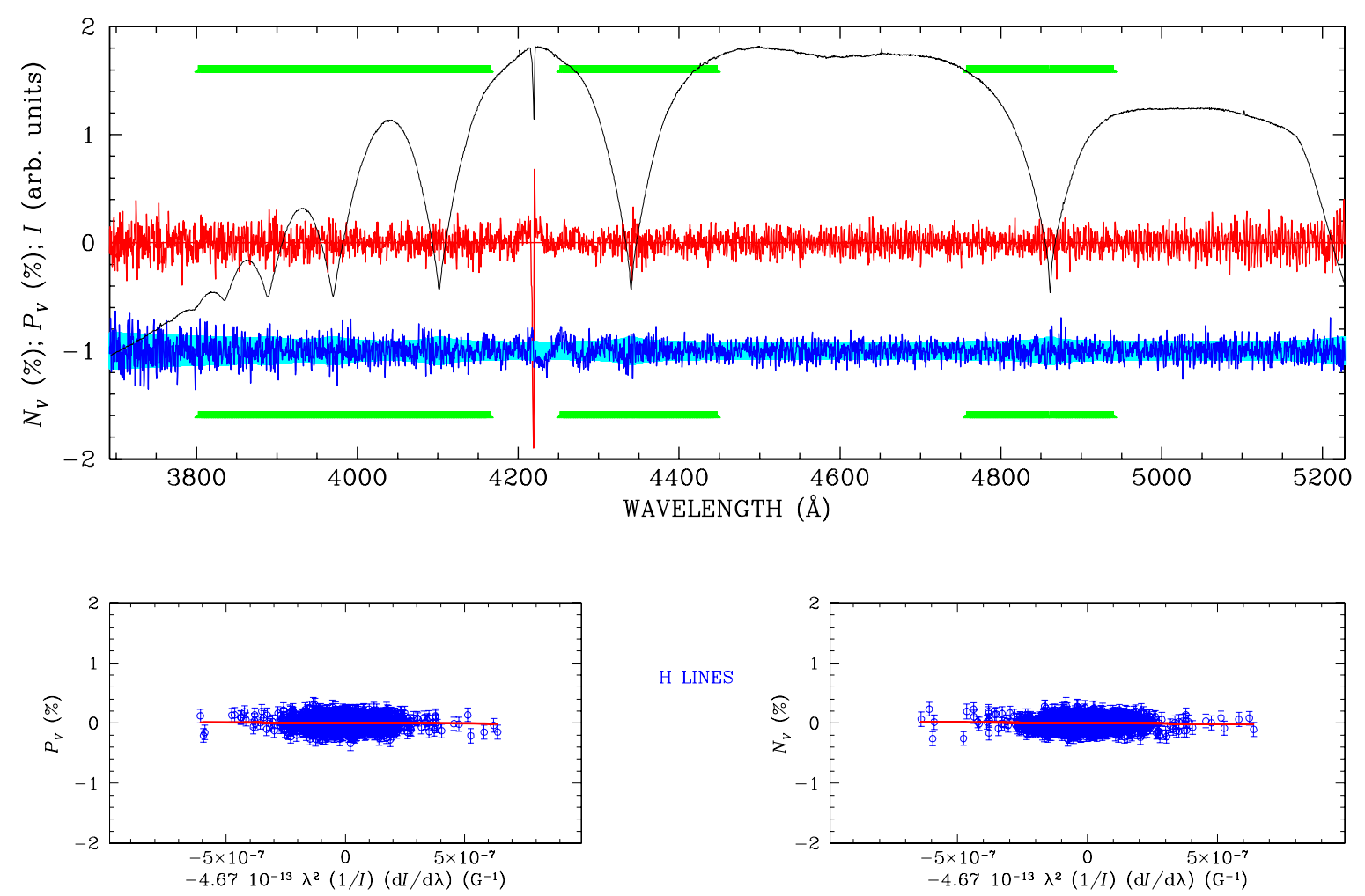

Fig. 4. Observation of 40 Eri B obtained with ISIS on MJD 57 056.92. The details shown in the figure are explained in the text. The $\left\langle B_{z}\right\rangle$ and $\left\langle N_{z}\right\rangle$ values deduced from the correlation diagrams in the lower part of the figure are $158 \pm 204 \mathrm{G}$ and $-254 \pm 186 \mathrm{G}$, respectively.

Table 4. Magnetic field measurements of 40 Eri B made with the ISIS medium-resolution spectrograph at the WHT.

\begin{tabular}{ccccc}
\hline \hline $\begin{array}{c}\text { Date } \\
\text { yr-mo-day }\end{array}$ & MJD & $\begin{array}{c}t_{\exp } \\
(\mathrm{s})\end{array}$ & $\begin{array}{c}\left\langle B_{z}\right\rangle \\
\text { (Gauss) }\end{array}$ & $\begin{array}{c}\left\langle N_{z}\right\rangle \\
\text { (Gauss) }\end{array}$ \\
\hline $2015-02-02$ & 57055.89 & 4000 & $199 \pm 393$ & $91 \pm 391$ \\
$2015-02-03$ & 57056.92 & 2800 & $158 \pm 204$ & $-254 \pm 186$ \\
$2015-02-04$ & 57057.95 & 3200 & $574 \pm 258$ & $184 \pm 261$ \\
\hline
\end{tabular}

present in bright DA white dwarfs in the temperature range of 8000-20000 K. We can make a useful comparison by computing the integration times that would be needed with these instruments to reach a given uncertainty in $\left\langle B_{z}\right\rangle$ for a given star. Since the brightest white dwarfs have $V$ magnitudes in the range of 12-13, an interesting comparison is to use the data reported above to estimate the integration times that would be required with various instruments to obtain measurement uncertainties of $500 \mathrm{G}$ for a star like 40 Eri B (i.e. a star with a similar $T_{\text {eff }}$ ) which has a $V$ magnitude of 12.5 .

This comparison is shown in Table 5. The values for the three spectropolarimeters discussed above are scaled directly from the most precise measurement of 40 Eri B reported for each instrument (the measurement which reaches the highest ratio of $\mathrm{S} / \mathrm{N}$ to the square root of the integration time). We use the data summarised by Landstreet et al. (2012) to include FORS1 in the table, using the best of three measurements of WD2149+021, a white dwarf with almost exactly the same $T_{\text {eff }}$ as 40 Eri B.

This table shows clearly that for the specific goal of detecting the weakest possible magnetic field in DA3-DA6 type white dwarf, the best choices are the high-resolution $\mathrm{H} \alpha$ method and conventional spectropolarimetry using FORS in the blue, with roughly similar integration times. Amazingly, the measurement
Table 5. Comparison of integration times $t_{\text {int }}$ required by each of the spectropolarimeters discussed above, and of ESO FORS, to reach $\sigma_{\left\langle B_{z}\right\rangle}=500 \mathrm{G}$ for an observation of a DA3 star of $V=12.5$.

\begin{tabular}{lr}
\hline \hline Spectropolarimeter & $\begin{array}{r}t_{\exp } \\
(\mathrm{s})\end{array}$ \\
\hline CFHT ESPaDOnS H $\alpha$ & 1500 \\
SAO MSS R15000 H $\beta$ & 19200 \\
WHT ISIS blue arm R600B grating & 7400 \\
ESO FORS grism 600B, 3470-5890 A & 1950 \\
\hline
\end{tabular}

would apparently take about the same amount of time with ESPaDOnS on the 3.6-m CFH Telescope as with FORS on an 8-m telescope. An efficient high-resolution spectropolarimeter on an 8-m telescope would require about $300 \mathrm{~s}$ of sky time to carry out this standard comparison measurement.

ISIS has about the same global efficiency as FORS, but has only about $1 / 4$ the telescope mirror area, which increases the required exposure times by about factor of four relative to a measurement with FORS. For our measurements, the SAO MSS spectropolarimeter has not been used in an optimal mode; the best measurement is the one made with R15000 but the efficiency would probably have been considerably higher with $\mathrm{H} \alpha$. Efforts are being made to bring this mode into convenient operation. In the meantime, the SAO MSS spectropolarimeter is best used for measurements of stars in which the magnitude of $\left\langle B_{z}\right\rangle$ reaches at least $20-30 \mathrm{kG}$.

For hotter DA1-DA2 white dwarfs in which the deep $\mathrm{H} \alpha$ core is weak or absent, so that the $\mathrm{H} \alpha$ method with a medium- or high-resolution spectropolarimeter is not effective, the best choices at present for the deepest field surveys are FORS in the southern sky and ISIS in the north. 


\section{Conclusions}

Known magnetic fields in white dwarf stars range in field strength from a few $\mathrm{kG}$ to several hundred $\mathrm{MG}$, an impressive five orders of magnitude. This fact, and the more detailed distribution of magnetic field strength and field structure, surely contain important clues about the origin and evolution of the observed, usually fairly simple, fossil fields.

Over this distribution of field strength, the most poorly studied section is the weak field end. White dwarfs with $\left\langle B_{z}\right\rangle$ of a few $\mathrm{kG}$ are rare (only three or four are known) and the known stars have not been extensively studied. We are engaged in filling in gaps in our knowledge of such stars.

The biggest obstacles to finding and studying the weakest field white dwarfs come from their general faintness (almost all fainter than $V=12$ ) combined with the extraordinary breadth of the few spectral lines. In the commonest white dwarfs, with H-rich atmospheres (DA stars) the Balmer lines can be $200 \AA$ wide. Such broad lines yield only a very weak polarisation signal when the field is a few $\mathrm{kG}$ in strength.

We have proposed studying the fields of DA stars in the temperature range of at least $8000-20000 \mathrm{~K}$ using the sharp and deep line core of $\mathrm{H} \alpha$, which in main sequence stars yields clearly recognisable polarisation signatures with fields of a few hundred G. We have tested the usefulness of this field measurement method by observing the bright DA white dwarf 40 Eri B, in which a variable field of a few $\mathrm{kG}$ has been reported (Fabrika et al. 2003). The star was observed repeatedly with the highresolution $(R=65000)$ ESPaDOnS spectropolarimeter at the CFHT. Reduction of the data showed that indeed field measurement using the core of $\mathrm{H} \alpha$ is very sensitive (measurements of $\left\langle B_{z}\right\rangle$ with uncertainties $\sigma_{\left\langle B_{z}\right\rangle}$ as low as $83 \mathrm{G}$ were obtained in one-hour integrations), but no trace of a magnetic field was found (Table 2). We conclude that $\left\langle B_{z}\right\rangle$ was almost certainly below $250 \mathrm{G}$ at the times when we observed 40 Eri B.

Measurements of the $\left\langle B_{z}\right\rangle$ values of 40 Eri B were also obtained with two other spectropolarimeters, the MSS of the SAO, and ISIS at the WHT (Tables 3 and 4), to validate the results found with ESPaDOnS. These measurements confirm the lack of a detectable field in 40 Eri B at the $2-3 \mathrm{kG}$ level. The observations also help to evaluate the important gain in field sensitivity realised with the $\mathrm{H} \alpha$ Zeeman measurements relative to the sensitivity achieved with normal low-resolution spectropolarimeters. The field measurements using $\mathrm{H} \alpha$ reached uncertainties more than a factor of two smaller than were obtained with the other instruments.

This comparison was extended by using the observational data from this paper, together with published white dwarf field measurements made with the ESO FORS low-resolution spectropolarimeter, to estimate the integration time needed to make a measurement of $\left\langle B_{z}\right\rangle$ accurate to $\sigma_{\left\langle B_{z}\right\rangle}=500 \mathrm{G}$ on a star similar to 40 Eri $\mathrm{B}$, but of magnitude $V=12.5$. It is found that the integration time required with ESPaDOnS (on a 3.6-m telescope) using only $\mathrm{H} \alpha$ is actually a little shorter than the time needed with FORS (on an 8-m telescope). The high-resolution measurement is clearly substantially more efficient than low-resolution observations on telescopes of the 4-m class for white dwarfs with suitable $\mathrm{H} \alpha$ lines.
The next step is to use this new technique to survey the brightest cool and intermediate DA white dwarfs of the northern hemisphere in order to identify a few more of the extremely weak-field white dwarfs, and to study the structure of their magnetic fields.

Acknowledgements. J.D.L. acknowledges ongoing financial support from the Natural Sciences and Engineering Research Council of Canada. D.G., E.S. and G.G. acknowledge the Russian Scientific Foundation (grant N14-5000043); GV acknowledges the Russian Foundation for Basic Research (RFBR grant N15-02-05183)

\section{References}

Angel, J. R. P., \& Landstreet, J. D. 1970a, ApJ, 162, L61

Angel, J. R. P., \& Landstreet, J. D. 1970b, ApJ, 160, L147

Angel, J. R. P., \& Landstreet, J. D. 1971, ApJ, 164, L15

Aznar Cuadrado, R., Jordan, S., Napiwotzki, R., et al. 2004, A\&A, 423, 1081

Bagnulo, S., Szeifert, T., Wade, G. A., Landstreet, J. D., \& Mathys, G. 2002, A\&A, 389, 191

Bagnulo, S., Landstreet, J. D., Fossati, L., \& Kochukhov, O. 2012, A\&A, 538, A129

Brinkworth, C. S., Burleigh, M. R., Lawrie, K., Marsh, T. R., \& Knigge, C. 2013, ApJ, 773, 47

Chountonov, G. A. 2004, in Magnetic Stars, eds. N. Arkhyz, Y. V. Glagolevskij, I. I. Kudryavtsev, \& I. I. Romanyuk, 286

Donati, J.-F., Semel, M., Carter, B. D., Rees, D. E., \& Collier Cameron, A. 1997, MNRAS, 291, 658

Fabrika, S., \& Valyavin, G. 1999, in 11th European Workshop on White Dwarfs, eds. S.-E. Solheim, \& E. G. Meistas, ASP Conf. Ser., 169, 225

Fabrika, S. N., Valyavin, G. G., \& Burlakova, T. E. 2003, Astron. Lett., 29, 737

Giammichele, N., Bergeron, P., \& Dufour, P. 2012, ApJS, 199, 29

Heber, U., Napiwotzki, R., \& Reid, I. N. 1997, A\&A, 323, 819

Joshi, S., Semenko, E., Martinez, P., et al. 2012, MNRAS, 424, 2002

Kawka, A., \& Vennes, S. 2012, MNRAS, 425, 1394

Kawka, A., Vennes, S., Schmidt, G. D., Wickramasinghe, D. T., \& Koch, R. 2007, ApJ, 654, 499

Kemp, J. C., Swedlund, J. B., Landstreet, J. D., \& Angel, J. R. P. 1970, ApJ, 161, L77

Kepler, S. O., Pelisoli, I., Jordan, S., et al. 2013, MNRAS, 429, 2934

Klochkova, V. G., Yermakov, S. V., \& Panchuk, V. E. 1996, Bulletin of the Special Astrophysics Observatory, 41, 58

Koester, D., Dreizler, S., Weidemann, V., \& Allard, N. F. 1998, A\&A, 338, 612

Koester, D., Napiwotzki, R., Christlieb, N., et al. 2001, A\&A, 378, 556

Koester, D., Voss, B., Napiwotzki, R., et al. 2009, A\&A, 505, 441

Kudryavtsev, D. O., Romanyuk, I. I., Elkin, V. G., \& Paunzen, E. 2006, MNRAS, 372,1804

Landstreet, J. D. 1992, A\&ARv, 4, 35

Landstreet, J. D., Silaj, J., Andretta, V., et al. 2008, A\&A, 481, 465

Landstreet, J. D., Bagnulo, S., Valyavin, G. G., et al. 2012, A\&A, 545, A30

Landstreet, J. D., Bagnulo, S., \& Fossati, L. 2014, A\&A, 572, A113

Liebert, J., Bergeron, P., \& Holberg, J. B. 2003, AJ, 125, 348

Monin, D. N., Fabrika, S. N., \& Valyavin, G. G. 2002, A\&A, 396, 131

Scargle, J. D. 1989, ApJ, 343, 874

Schmidt, G. D. 2001, in Magnetic Fields Across the Hertzsprung-Russell Diagram, eds. G. Mathys, S. K. Solanki, \& D. T. Wickramasinghe, ASP Conf. Ser., 248, 443

Tout, C. A., Wickramasinghe, D. T., Liebert, J., Ferrario, L., \& Pringle, J. E. 2008, MNRAS, 387, 897

Valyavin, G., \& Fabrika, S. 1999, in 11th European Workshop on White Dwarfs, eds. S.-E. Solheim, \& E. G. Meistas, ASP Conf. Ser., 169, 206

Valyavin, G., Bagnulo, S., Monin, D., et al. 2005, A\&A, 439, 1099

Vanlandingham, K. M., Schmidt, G. D., Eisenstein, D. J., et al. 2005, AJ, 130, 734

Wickramasinghe, D. 2001, in Magnetic Fields Across the Hertzsprung-Russell Diagram, eds. G. Mathys, S. K. Solanki, \& D. T. Wickramasinghe, ASP Conf. Ser., 248, 453

Wickramasinghe, D. T., Tout, C. A., \& Ferrario, L. 2014, MNRAS, 437, 675 